\title{
Link and Cost Optimization of FTTH Network Implementation through GPON Technology
}

\author{
Dheyaa Jasim Kadhim, Nahla Abdulrahman Hussain \\ Electrical Engineering Department, University of Baghdad, Baghdad, Iraq \\ Email: dheyaajk@gmail.com \\ Received May 2013
}

\begin{abstract}
The motivation behind this work is the need for performance analysis of Al-Gehad/Baghdad "Fiber To The Home" (FTTH) network which is one of the Iraq Telecommunication Post and Company (ITPC) FTTH projects in 2010 in Baghdad City. Based on the detailed study of this project, the design, implementation, and analysis of this network has been putting under consideration through a mathematical model simulated and performance evaluated. The link utilization and number of OLT ports (PON) had been studied and evaluated as a function of cycle time. Cost optimization was done through evaluation the total cost of PON system and who it affected with the number of ONUs (i.e. splitting ratio), as well as cost ratio between EPON and GPON is evaluated. Then GPON system has been optimized to analyze and evaluate the feeder network cost, distribution network cost, and total network cost.
\end{abstract}

Keywords: Gigabit Passive Optical Network (GPON); Fiber to the Home (FTTH) Network; OLT and ONU

\section{Introduction}

With the development of many advanced multimedia applications, there is a massive increase in bandwidth demand. PON technology, is featured with flexible bandwidth, good management function and high transmission efficiency, has already been widely used in access network [1]. From 2003, the International Telecommunication Union-Telecommunication Standardization Sector (ITU-T) has published G.984.x standard to define the GPON system. GPON is a Passive Optical Network (PON) operating at $2.488 \mathrm{Gbps}$ downstream and $1.244 \mathrm{Gbps}$ upstream rates [2].

FTTH (Fiber to the Home) is a viable solution to deploy access network, which allows serving multiple end users simultaneously at high speed. In the common architecture, FTTH networks carry signal from the Optical Line Terminal (OLT) usually placed in the center office, to end user devices (ONT) by running a fiber from this center office to the remote node in the middle of the way and then continuing by different fibers to different customers. For network access, it is always desired that fiber optic network connectivity be the major area of cost minimization, while keeping quality of service as high as possible. This is because overall cost of setting up a fiber optic cable route is usually very high in relation to other components of the network.

In [3] a modeling of the capital investments and operations expenses incurred for some operator cases, and identifies the key parameters that impact FTTH economics covering these major technologies. In [4] a comprehensive cost modeling of FTTH was presented, which includes outside plan, head end, and premises equipment and labor with enhanced reach and split ratio. A linear programming in generating a model to solve the access network problem of connecting buildings across the university is found in [5].

In this work, we will introduce study, design, implementation of Al-Gehad/Baghdad FTTH network, then a performance analysis of this network was done through evaluating link utilization and number of OLT ports (PON) as a function of cycle time. Cost optimization was done through evaluation the total cost of PON system and who it affected with the number of ONUs (i.e splitting ratio), as well as cost ratio between EPON and GPON is evaluated. Then GPON system has been optimized to analyze end evaluate the feeder network cost, distribution network cost, and total network cost.

The rest of this paper is structured as follows; Section 2 will illustrate the project information, the network topology network will be explained in Section 3 and its performance analysis is illustrated in Section 4, while its numerical results are shown in Section 5. Conclusion will be made in Section 6 .

\section{Optical Network Modeling}

Iraq Telecommunication and Post Company (ITPC), fo- 
cuses on the users' requirements and the technology trends, is constructing the national FTTH network to provide Voice over IP (VoIP) and High Speed Internet (HSI) services to the residential customers. FTTH network in AlGehad consists from two OLTs placed in Al-Gehad exchange will be serving 6000 subscribers in total area of about $32 \mathrm{~km}$. It has been planned to use 6000 ONTs, one ONT per subscriber. Each OLT will connect to the converged Layer 3 switches and then the routers to ITPC Passive Distribution Network (PDN) network. The length of feeder cables is $243 \mathrm{~km}$, and the length of distribution cables is $405 \mathrm{~km}$, so the total fiber cables length will be $647 \mathrm{~km}$.

After studying the project maps and summarized the varying building features and application scenarios of the detail site survey, and based on the requirement of IPTC, the design will be as follow [6]:

- For general network topology, a ring topology is used to deploy the FTTH passive network, offer the protection to Optical Distribution Network (ODN).

- There is $20 \%$ of spare capacity in the feeder cables, from the Central Office up to the Fiber Distribution Terminal (FDT), to meet the future requirements.

- Feeder section from CO to FDT, $144 \mathrm{~F}$ optical cable will be proposed by ring topology and 2:4 first level splitters will be proposed in FDT cabinet.

- Distribution section from FDT to Fiber Access Terminal (FAT), 72F, 48F, 24F, and 12F optical cable will be proposed by star topology, 1:16 second level splitter will be proposed in FAT, which is closure to wall mounted FAT product to meet different requirement and scenarios.

Two topologies are used as shown in Figure 1, ring topology for feeder cables that are connect the Fiber Distribution Terminals (FDTs) to the Central Office (CO) by first level splitting of 2:4, and star topology to connect several numbers of Fiber Allocation Terminal (FAT) to each FDT by second level splitting of 1:16.

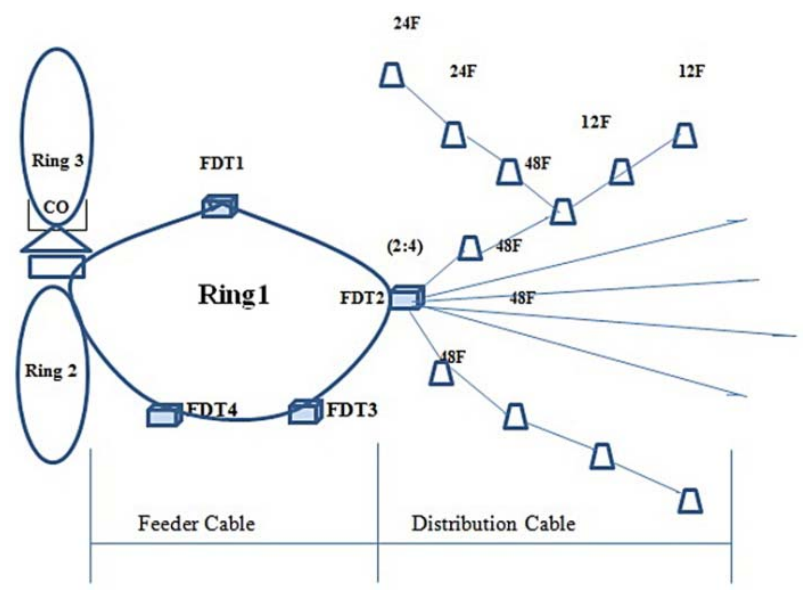

Figure 1. Network Topology.
OLT will be deployed in each central office and the quantity will be calculated to satisfy the coverage capacity. Each OLT will connect to the converged Layer 3 switches and then the routers to ITPC PDN network. BRAS will be installed in the core central offices. BRAS are full-loaded configuration with the processing capability of not less than 115,000 lines. In the normal status, each BRAS just take the service traffic which belongs to the corresponding areas. When one is broken, the other BRAS will take the whole service. AAA is the same with BRAS. EMS remote redundancy is ensuring the reliability.

For FTTH solution the access layer will provide OLT equipment in exchange; all subscribers will be connected through Optical Distribution Network (ODN). The proposed connection will be from the OLT to access point Fiber Access Terminal distribution box (FAT).

As it is obvious from Figure 2 the FTTH system comprises the Optical Line Terminal (OLT) on the CO, the Optical Network Terminal (ONT) on the user side, and the Optical Distribution Network (ODN). The FTTH construction features the PON technology, which provides point to point and point to multi-point applications. ODN provides the physical channels from OLT and ONT to communicate with each other.

\section{Performance Analysis of FTTH Network}

Use of passive optical networks is very advantageous in designing FTTH architectures [7]. If a two main standards (EPON and GPON), named XPON, are considered, a series of important optimization problems for the design, plan, and deployment of FTTH networks and passive optical networks should be considered since they truly effect on the network efficiency and performance.

The target is to compare the network cost of an EPON and GPON system based on the utilization of the optical link's transport capacity. The utilization affects directly the segmentation need in an optical network and this effect on the total network cost [8].

\subsection{Link Utilization}

Equation (11) calculates link utilization $\left(v_{\mathrm{Ed}}\right)$ of an EPON downstream channel.

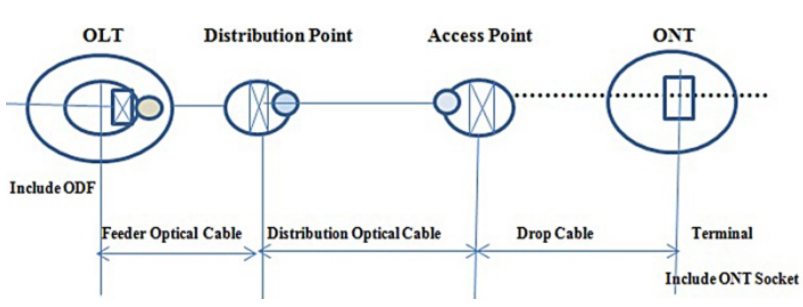

Figure 2. General FTTH System. 


$$
v_{\mathrm{Ed}}=\frac{\frac{f_{E}}{f_{E o}+f_{E}}\left[t_{c} * B_{E}-N_{O N U} * f_{c m}\right]}{t_{c} * B_{E}}
$$

Where $f_{E}$ is the EPON frame payload, $f_{E o}$ the EPON frame overhead, it is taken to be 42 bytes, $B_{E}$ the bit rate of an EPON link, it is equal to $1.25 \mathrm{Gbps}$, $N_{O N U}$ the ONUs number in the network segment which is taken $10,000, f_{c m}$ the length of control message, it is equal to 88 bytes, and $t_{c}$ the cycle time. Utilization $\left(v_{\mathrm{EU}}\right)$ of an EPON is given by Equation (2);

$$
v_{\mathrm{Eu}}=\frac{\frac{f_{E}}{f_{E o}+f_{E}}\left[t_{c} * B_{E}-N_{O N U}\left(f_{c m}+t_{p o} * B_{E}\right)\right]}{t_{c} * B_{E}}
$$

Where $t_{p o}$ is the physical layer overhead (i.e. guard band), it is taken $1.44 \mu \mathrm{sec}$. Utilization $\left(v_{\mathrm{Gd}}\right)$ of an GPON downstream is given by Equation (3);

$$
v_{\mathrm{Gd}}=\frac{\frac{f_{E p}}{f_{G E M o}+f_{E p}}\left[t_{d} * B_{G}-f_{G o} ? f_{a o}\left[\frac{N_{O N U^{*} t d}}{t_{c}}\right]\right]}{t_{d} * B_{G}}
$$

Where $f_{\text {GEMo }}$ is the GEM framing overhead for Ethernet payload which is equal 30 bytes, $f_{E P}$ is Ethernet payload, $t_{d}$ is GPON duration of downstream frame, its equal $125 \mu \mathrm{sec}, B_{G}$ the GPON bit rate $(1.25 \mathrm{Gbps})$, $f_{G o}$ (27 bytes) is the GPON downstream frame overhead, and $f_{a o}$ (27 bytes)is the upstream allocation overhead. Utilization $\left(v_{\mathrm{Gu}}\right)$ of an GPON upstream is given by (4);

$$
v_{\mathrm{Gu}}=\frac{\frac{f_{E p}}{f_{G E M o}+f_{E p}}\left[t_{d} * B_{G}-\left[\frac{N_{O N U * t_{d}}}{t_{c}}\right] f_{\text {plou }}+\overline{f_{d b r u}}\right]}{t_{d} * B_{G}}
$$

Where $f_{\text {plou }}$ is the length of physical layer overhead (include PLOAMu field) it taken 15 bytes, and $f_{d b r}$ is the average number of DBRu fields in an upstream GPON frame.Since an ONU can send several GEM frames during its time slot and only the first of them carries the PLOu field and all frames carry the DBRu field, $\overline{f_{d b r}}$ is approximated by:

$$
\overline{f_{d b r u}}=\frac{\frac{t_{c^{*} B_{G}}}{N_{O N U}}-f_{\text {plou }}}{f_{\text {dbru }+f_{G E M}}}
$$

Where $f_{G E M}=f_{G E M o}+f_{E P}$

\subsection{Network Segmentation}

At building a passive optical network, segmentation is the way to guarantee fair transport capacity per subscriber as shown in Figure 3.

A number of needed network segments are determined

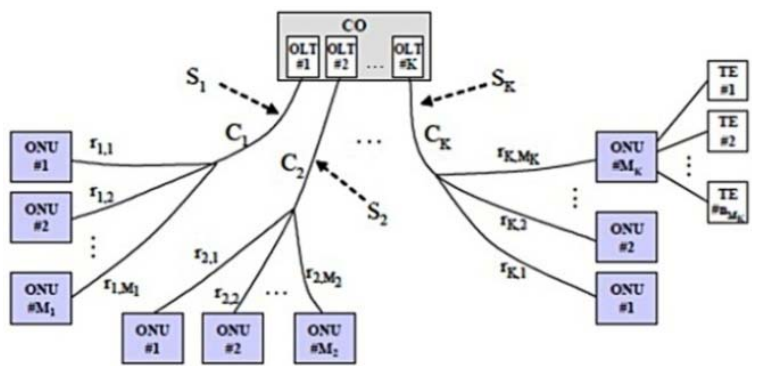

Figure 3. Segmented PON layout [10].

according to the total transport capacity and number of subscribers. For both EPON and GPON systems, assume that the total transport capacity of segment $\operatorname{Seg}_{\mathrm{k}}$ is $\mathrm{C}_{\mathrm{k}}$, line coding efficiency is $\sigma$ and utilization of the transport channel capacity is $v$, so $\mathrm{B}_{\mathrm{k}}$ the total bit rate available for user in segment $\mathrm{Seg}_{\mathrm{k}}$ is:

$$
\mathrm{B}_{\mathrm{k}}=\sigma v \mathrm{C}_{\mathrm{k}}
$$

The total available bit rate for the segment $\mathrm{Seg}_{\mathrm{k}}$ is the sum of the traffic of all ONUs connected to it as below:

$$
\mathrm{B}_{\mathrm{k}}=\sum_{i=0}^{M k} \mathrm{~b}_{\mathrm{k}, \mathrm{I}}
$$

Where $\mathrm{M}_{\mathrm{k}}$ represents the number of ONUs connected to the ith port of the OLT. Thus, the number of required segments (OLT ports) will be [10]:

$$
\mathrm{K}=\frac{\Omega \beta \mathrm{N} r_{o}}{\sigma v \mathrm{C}}
$$

Where $\Omega$ is a broadband access (take rate) as $(0<\Omega<$ $1), \beta$ is the percentage of active subscribers that operate during a busy hour, $\mathrm{N}$ is ONUs number, $r_{o}$ is the average bandwidth required to support all requested services on one or all OLT ports, $\sigma$ is line coding efficiency, $v$ is the utilization of the transport channel capacity, and $\mathrm{C}$ is transport capacity.

The number of PONs determines the number of OLTs required serving a whole region. This enables network planners to know the cost required for installing, configuring and upgrading GPON resources. Each PON can serve up to $\Omega \mathrm{N} / \mathrm{K}$ subscribers, which can be expressed as follows [10]:

$$
\text { Number of subscribers }=\frac{\Omega N}{K}=\frac{\sigma v \mathrm{C}}{\beta r_{o}}
$$

This formula indicates that the number of subscribers supported on a single OLT port (PON) depends mainly on $\mathrm{r}_{\mathrm{o}}$ and $\beta$.

Each OLT of Al-Gehad FTTH network has 18 ports, 14 are used and the other four ports are left for the possibility of traffic growth.

\subsection{Relative Network Cost}

The number of network segments is the most important 
factor when calculating the cost of EPON and GPON networks. The number of segments indicates the difference between an EPON and GPON networks in the amount of installing fiber and the number of the needed transceivers in the network. The relative network cost of an EPON and GPON is given by [8]:

$$
\varphi=\frac{\sigma_{G} v_{G C_{G}}}{\sigma_{E} v_{E C_{E}}}
$$

Where $\varphi=\mathrm{K}_{\mathrm{E}} / \mathrm{K}_{\mathrm{G}}$, and Setting the parameters $(\beta, \Omega, N$, and $r_{o}$ ) equal in both approaches, (i.e. EPON and GPON).

\section{GPON Capacity and Cost Optimization}

Optimization approach aims to optimally allocate the capacity on GPON access network links on Al-Gehad FTTH network, which can support current and future traffic demands, while guaranteeing a minimum throughput required for all class $j$ traffic, $\rho_{j}^{\min }$. This approach is expressed in an optimization problem as follows:

- Cost minimization of the capacity of the links in the GPON access network. Where $\alpha_{j}$ cost per bit with each link $l$ in the GPON access network, and $\mathrm{C}_{l}$ is the link capacity.

$$
\min \sum_{j \in l} \alpha_{j} \mathrm{C}_{1}
$$

- Capacity constraints of these links, where the total traffic load generated by all flows sharing a link should be less than its capacity,

$$
\mathcal{F}_{l}=\sum_{j \in H_{l}} \mathcal{F}_{j} \leq C_{l} .
$$

Subject to $\sum_{j \in H_{l}} \mathcal{F}_{j}<C_{l}, \forall l \in L$ Throughput performance constraint in a GPON access network, where the throughput of each class $\mathrm{j}$ traffic should be greater than the minimum required throughput of this class. Where $H_{l}$ denotes the set of flows that share link $l$.

$$
\frac{1}{\rho_{j}} \leq \frac{1}{\rho_{j}^{\text {min }}}, \forall \mathrm{j}=1,2, \ldots, \mathrm{Z}
$$

The throughput constraint can be expressed with respect to the average delay of generated traffic flows as follows: follows $\rho_{\mathrm{j}}=\frac{E\left[S_{j}\right]}{E\left[T_{j}\right]}$, where $S_{j}$ represent the volume of traffic flows. Thus, the throughput performance can be written as follows:

$$
\mathrm{E}\left[\mathrm{T}_{\mathrm{j}}\right] \leq \frac{E\left[S_{j}\right]}{\rho_{j}^{\min }}, \forall j=1,2, \ldots, N
$$

Where $\rho_{\mathrm{j}} \geq \rho_{j}^{\min }$.

After some algebraic manipulations, the optimal capacities of both GPON feeder link and distribution links can be given by [9]:

$$
\begin{gathered}
\mathrm{C}_{\text {feeder }}=\mathrm{N} \mathcal{F}+\rho^{\min }\left[1+\sqrt{N} \sqrt{\frac{\alpha_{d}}{\alpha_{f}}}\right] \\
\mathrm{C}_{\text {distribution }}=\mathcal{F}+\rho^{\min }\left[1+\frac{1}{\sqrt{N}} \sqrt{\frac{\alpha_{f}}{\alpha_{d}}}\right]
\end{gathered}
$$

Where $N$ is the number of ONUs, $\mathrm{C}_{\text {feeder }}$ is the feeder link capacity and $\mathrm{C}_{\text {distribution }}$ is the distribution links capacity. If we consider that the cost of transmitting traffic on feeder link is the same as that on a GPON distribution links, then $\alpha_{f}=\alpha_{d}$, and for simplicity take $\alpha_{f}=\alpha_{d}=1$. Then, as $\mathrm{N} \mathcal{F} \rightarrow \infty$, the optimal capacity of the GPON feeder link and distribution links respectively will be:

$$
\begin{gathered}
\mathrm{C}_{\text {feeder }}=\mathrm{N} \mathcal{F}+\rho^{\min } \\
\mathrm{C}_{\text {distribution }}=\mathcal{F}+\rho^{\min }
\end{gathered}
$$

So, the optimal total cost will be

$$
\mathrm{C}_{\text {feeder }}+\mathrm{N} \mathrm{C}_{\text {distribution }}=2 \mathrm{~N} \mathcal{F}+(\mathrm{N}+1) \rho^{\min }
$$

\section{Nnumerical Results}

The results and discussions included link utilization of xPON, number of PONs and network cost analysis. Figure 4 illustrates the utilization as a function of the cycle time in two cases when the payload size is 46 bytes and 1500 bytes for single and multi-OLT EPON and single OLT GPON systems. This Figure shows that utilization is proportional with cycle time (i.e. short cycle time means less link utilization), with short access. Downstream direction achieves better utilization than the upstream does, but when cycle time increases the difference will be very little. Lower utilization obtained with multi-OLT EPON network, this is because the increasing number of ONUs since the total bandwidth must be divided among larger number of ONUs [10]. For this reason, the second level splitting ratio in Al-Gehad FTTH network is 1:16 only.

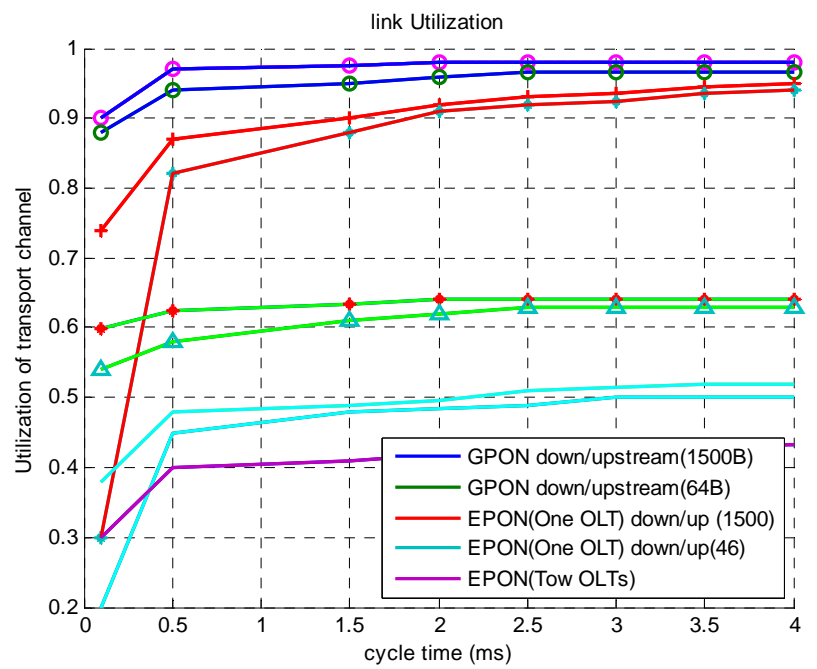

Figure 4. EPON and GPON Link Utilization vs. Cycle Time. 
Figure 5 shows that the number of PONs grows linearly with take rate. For the same type of traffic, GPON needs fewer segments than EPON. Multi-OLT EPON network requires larger number of segments (PONs) than single OLT XPON to assure services required to the larger number of subscribers.

Figure 6 illustrates the relationship between cycle time and cost ratio $(\varphi)$ for 46 and 1500 bytes payload. For small payload size and short cycle time, cast ratio is higher, this refers to that GPON fits better for low volume and small delay traffic for example voice over IP (VoIP) and PSTN. For this type of traffic and in upstream direction, $\varphi$ is about 1.7. Figure 7 shows the relationship between the capacity of the GPON link, $\mathrm{C}_{\mathrm{Feeder}} / \mathrm{N}$, and the number of ONUs (N) for different amount of minimum throughput, $\rho^{\min }$. Subscribers are allocated less bandwidth as number of ONU increases. This is due that the capacity of GPON link is dimensioned such that $\mathrm{C}_{\text {feeder }}$ equals the worst-case load $(\mathrm{MF})$. The optimum value of $\mathrm{C}_{\text {feeder }}$ converges around the total capacity required for accommodating general traffic load in addition to $\rho^{\min }$. Figure 8 shows the optimal distribution link capacity required to guarantee different requirements of throughput, $\rho^{\min }$. Each ONU can support different applications, therefore distribution links should have sufficient bandwidth such that the remaining capacity on distribution links, can guarantee the minimum throughput. Figure 9 illustrates the optimal total cost of GPON access network for different throughput requirements. The total costs converge in a slower manner for high minimum requirements, than when $\rho^{\min }$ is taken to be smaller value.

\section{Conclusions}

A study of ITPC access network project 2010 in Baghdad by taking Al-Gehad FTTH network as a study case has been done. A study of link utilization of channel capacity

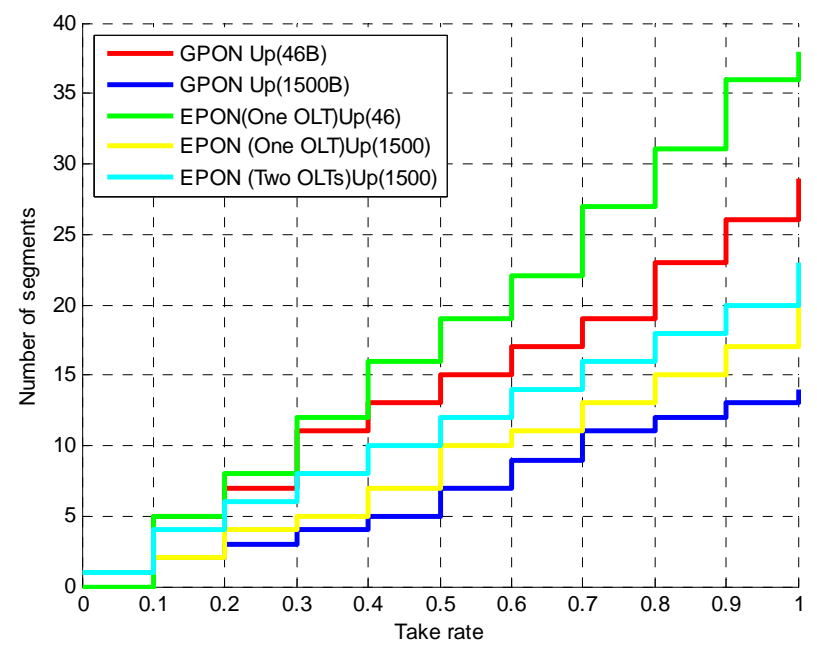

Figure 5. Number of PONs vs. Take rate.

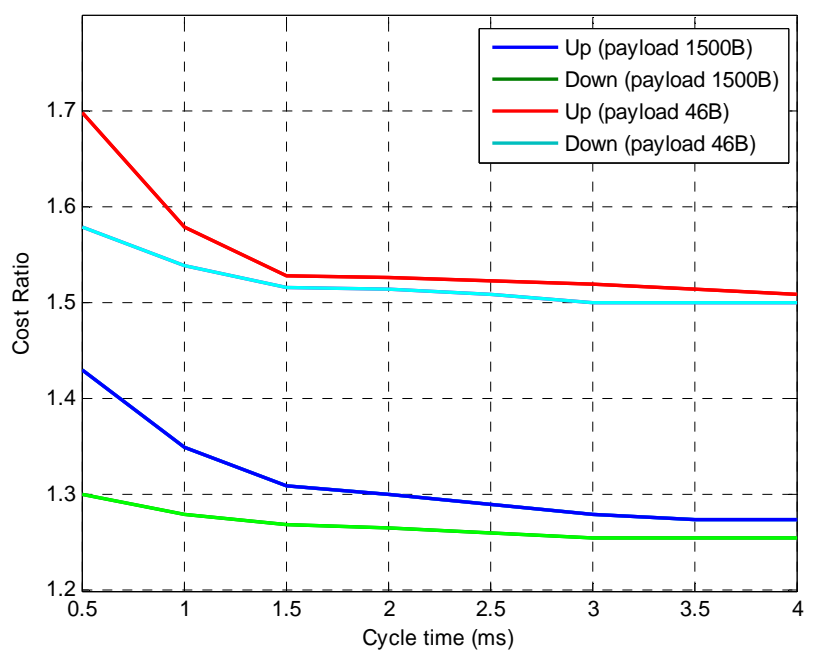

Figure 6. EPON-to-GPON Cost Ratio.

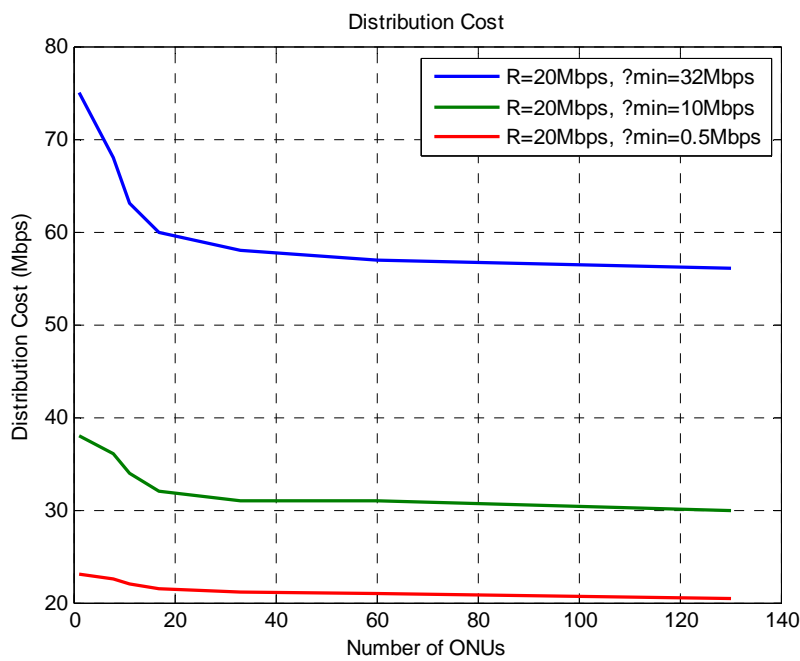

Figure 7. GPON Feeder Link Capacity vs. Number of ONUs.

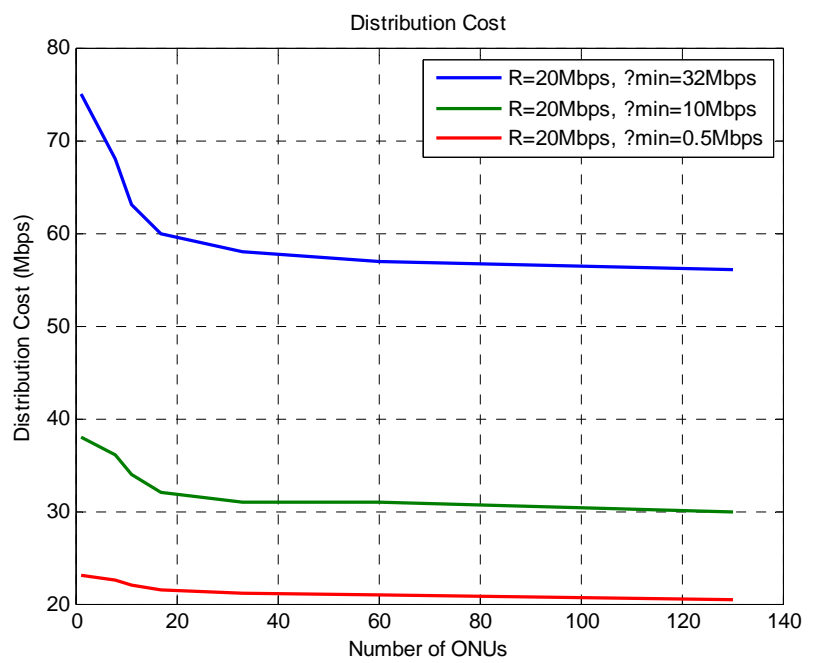

Figure 8. GPON Distribution Link Capacity vs. Number of ONUs. 


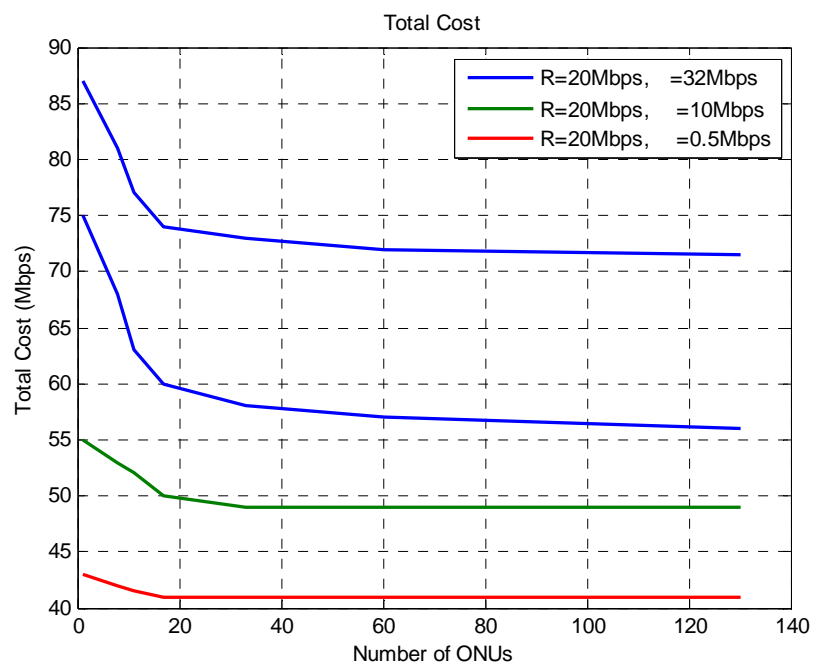

Figure 9. Total Cost of GPON Resources Vs. Number of ONUs.

in both EPON and GPON has been done and uses that information to compare the cost of these two systems. From the numerical results we can say that the GPON system uses the link capacity more efficiently than EPON system does. The cost to build an EPON or GPON system is almost the same, the relative cost is affected widely by the cost of transceivers. For example, for VoIP service, the GPON transceiver is about $70 \%$ more expensive than EPON transceivers.

In this work we can find that GPON network planners should take into their considerations the number of demanding and basic services that will be supported on each PON (OLT port) to achieve traffic balancing among all PONs. Link utilization can be used to calculate the number of subscribers that can be supported on a single PON and then can determine the size of population and services that can be supported on OLT ports.

An optimization problem has been formulated to find the optimal capacity and cost of GPON access network links guarantee that a minimum throughput can be ensured for supported traffic classes.

\section{REFERENCES}

[1] I. Cale, A. Salihovic, M. Ivekovic and T-HTd. D. Split, "Gigabit Passive Optical Network-GPON," 29th International Conference ITI 2007.

[2] S. Lallukka and P. Raatikainen, "Link Utilization and Comparison of EPON and GPON Access Network Cost," Proceeding of IEEE Globecome, 2005.

[3] S. Kulkarni, M. El-Sayed, P. Gagen and B. Polonsky, "FTTH Network Economics: Key Parameters Impacting Technllogy Decisions," Network Planning-Bell Labs, Alcatel-Lucent Technologies, 2010.

[4] M. Vaughn, D. Kozischek, D. Meis, A. Boskovic and R. Wagner, "Value of Reach and Split Ratio Increase in FTTH Access Network," Journal of Lightwaves Technology, Vol. 22, No. 11, 2004.

[5] C. Bolu, A. Talulade and A. Adeshina, "University Optical Fiber Network Access Optimization: A Case Study," International Journal of Mechanical \& Mechatronics Engineering, Vol. 12, No. 6, 2012.

[6] ITPC Documents, Reports, and Maps.

[7] D. Nowak, "Dynamic Bandwidth Allocation Algorithms for Differentiated Services enabled Ethernet Passive Optical Networks with Centralized Admission Control," Ph.D. Thesis, 2005.

[8] H. Alshaer and J. J. Elmirghani, "Enabling Novel Premium Service Classes in DiffServover MPLS-Enabled Network," International Journal of Network Management, Vol. 18, 2008, pp. 447-464. http://dx.doi.org/10.1002/nem.693

[9] M. D. Vaughn, D. Kozischek, D. Meis, A. Boskovic and R. E. Wanger, "Value of Reach-and-Split Ratio Increase in FTTH Access Networks," Journal of Lightwave Technology, Vol. 22, No. 11, 2004, pp. 2617-2622. http://dx.doi.org/10.1109/JLT.2004.836741

[10] S.ami Lallukka and P. Raatikainen, "Link Utilization and Comparison of EPON and GPON Access Network Cost," Proceedings of IEEE Conference on Global Telecommunications, 2005.

[11] H. Alshaer, R. Shubair and M. Alyafei, "A Framework for Resource Dimensioning in GPON Access Network," International Journal of Network Management, Vol. 22, No. 3, 2011, pp. 199-215. 